\title{
CLINICAL AND BIOCHEMICAL MANIFESTATIONS PRODUCED BY SCORPION (Hemiscorpius lepturus) VENOM IN EXPERIMENTAL ANIMALS
}

\author{
ZARE MIRAKABBADI A. (1), ZOLFAGHARIAN H. (1), \\ HEDAYAT A. (1), JALALI A. (2)
}

(1) Department of Venomous Animals and Antisera Production, Razi Vaccine and Serum Research Institute, Hesarak, Karaj, Iran; (2) Department of Pharmacology and Toxicology, School of Pharmacy, Jundishapur University, Ahvaz, Iran.

ABSTRACT: Several studies have been published about the clinical and biochemical manifestations produced by the venom of scorpions of the Buthidae family, but very few reports have indicated the manifestations induced by the venom of the Scorpionidae family. Hemiscorpius lepturus is an important scorpion species present in the south and southwestern part of Iran, causing morbidity and mortality in children and adults. For the present study, $H$. lepturus venom was extracted by electric shock and subcutaneously injected $(6.3 \mathrm{mg} / \mathrm{kg})$ into a group of six rabbits. Blood collection was carried out before and three hours after venom injection for determination of osmotic fragility and levels of blood sugar, alanine aminotransferase (ALT), aspartate aminotransferase (AST), lactate dehydrogenase (LDH), creatine phosphokinase $(\mathrm{CPK})$ and alkaline phosphatase (ALP). In vitro studies were also carried out to verify the osmotic fragility of red blood cells (RBCs) exposed to venom concentrations ranging from $0-90 \mu \mathrm{g} / 2 \mathrm{ml}$ blood. Results showed the extreme effect of this venom on the lysis of RBCs both in vitro and in vivo. Venom injection caused significant $(p>0.001)$ increase in ALT, AST, LDH and blood sugar levels. There was also an increase in CPK, and ALP levels after venom injection; however, it was not statistically significant. All animals died four hours after having received the venom. The current study revealed that the neurological effect of $H$. lepturus venom is similar to that of scorpions of the Buthidae family. However, they differ in RBCs lysis, which was highly significant when induced by $H$. lepturus venom, probably due to the presence of a type of phospholipase in this venom. Further studies are needed to provide a clearer view of the mechanism of action of $H$. lepturus venom.

KEY WORDS: Hemiscorpius lepturus, scorpions, osmotic fragility, Iran, biochemical changes.

CONFLICTS OF INTEREST: There is no conflict.

\section{CORRESPONDENCE TO:}

ABBAS ZARE MIRAKABBADI, Department of Venomous Animals and Antisera Production, Razi Vaccine and Serum Research Institute, Hesarak, Karaj, Iran. Email: zare@zare.ir. 


\section{INTRODUCTION}

Venomous scorpions pose a great health hazard to children and adults in the southwestern part of Iran $(1,14)$, where the most common scorpion genus is Hemiscorpius. Although $H$. lepturus belongs to the Scorpionidae family and its venom gland is very small, it can cause death to both children and adults $(1,5,14)$. Many studies have reported manifestations caused by venoms of scorpions of the Buthidae family $(3,10,17,18)$ but very few have indicated the signs and symptoms as well as the serum biochemical changes induced by $H$. lepturus venom (14).

The most significant manifestation in envenomation by $H$. lepturus is hemolysis. Approximately $40 \%$ of the stung patients need hemodialysis and, sometimes, packed cells treatment (14). The mechanism of death caused by $H$. lepturus envenomation is thought to be quite different from that caused by scorpions of the Buthidae family; clinicians in the south of Iran believe that toxin distribution rate of $H$. lepturus venom is very slow (14). Thus, the current study aimed at investigating cellular and biochemical alterations caused by $H$. lepturus envenomation and at verifying whether its mechanism of manifestations is quite different from or similar to that of scorpions of the Buthidae family.

\section{MATERIALS AND METHODS}

Five thousand scorpions ( $H$. lepturus) were collected from the southwestern part of Iran and identified by experts at Razi Vaccine and Serum Research Institute, Iran. Venom was milked by electric shock, lyophilized and preserved until used. The venom LD $_{50}$ was determined in mice $(18-20 \mathrm{~g})$ by the method of Reed and Muench (15).

Six male healthy rabbits of $1.5 \pm 0.2 \mathrm{~kg}$ were selected for this experiment. The venom $(6.3 \mathrm{mg} / \mathrm{kg}$ of body weight) was subcutaneously injected. Blood sampling was carried out via a polyethylene cannula (G21) inserted into the marginal ear vein of each overnight-fasted animal before and three hours after venom injection. Part of the collected blood was poured into heparinized tubes for osmotic fragility tests and part of it underwent serum separation for analysis of blood sugar, ALT, AST, LDH, CPK and ALP levels (kits supplied by Sigma®). 
In vivo osmotic fragility test was carried out with whole blood collected before and three hours after venom injection; blood samples were exposed to various $\mathrm{NaCl}$ concentrations (from 0 to $0.9 \%$ ) and incubated for $30 \mathrm{~min}$ at $37{ }^{\circ} \mathrm{C}(13)$.

For in vitro osmotic fragility test, $2 \mathrm{ml}$ of normal whole blood was incubated at various concentrations of $\mathrm{H}$. lepturus venom $(1,10,30,60$ and $90 \mu \mathrm{g})$ for $30 \mathrm{~min}$ at $37^{\circ} \mathrm{C}$; then, samples were exposed to $\mathrm{NaCl}$ concentrations ranging from 0 to $0.9 \%$ for $30 \mathrm{~min}$ at $37{ }^{\circ} \mathrm{C}(13,16)$.

\section{RESULTS}

The venom $L D_{50}$, determined in mice, was $126 \mu \mathrm{g} / \mathrm{mouse}$. After venom injection $(6.3 \mathrm{mg} / \mathrm{kg})$, animals were observed for signs and symptoms during the whole experiment. Within $20-35 \mathrm{~min}$ after venom administration, all animals started to present signs of envenomation like edema at the injection site, increased heartbeat rate, salivation and lacrimation, and altered breathing rhythm. Although urination and change in the urine color from light yellow to red were expected, no such alterations were observed in the experimentally envenomed rabbits during the experiment. Edema at the injection site was measured and characterized by a $1-2 \mathrm{~cm}$ swollen red patch. In two rabbits, the swollen red patch was extended to $8-10 \mathrm{~cm}$. Within two hours after injection, all animals showed wheezing and coughing. No hematuria or dysentery was observed in envenomed rabbits. Two hours after venom injection, all animals showed muscle contraction and paralysis. They could not stand on their feet. Death occurred as a consequence of increased heartbeat rate, difficult breathing and complete feet paralysis, similarly to cyanide poisoning.

Figure 1 shows the osmotic fragility of RBCs, normal and exposed to various concentrations of $H$. lepturus venom. Hemolysis rate increased with increasing concentrations of venom. The figure clearly shows a significant $(p<0.001)$ shift to the right as the venom concentration increased. Changes in the osmotic fragility of RBCs exposed to $1 \mu \mathrm{g}$ venom were insignificant, compared with control.

Figure 2 shows the comparison of osmotic fragility of RBCs from rabbits' blood samples before and after venom injection. The figure clearly shows a significant shift to the right, which indicates an increased osmotic fragility of RBCs exposed to $H$. lepturus venom. 
Table 1 shows the levels of serum enzymes ALT, AST, LDH, CPK and ALP as well as blood sugar. There was a highly significant $(p<0.001)$ increase in the concentration of LDH, ALT and AST in the serum of rabbits three hours after venom injection. Although there was also an increase in CPK and ALP levels after venom injection, it was not significant when compared with the levels of these enzymes before injection. Blood sugar also significantly $(p<0.001)$ increased after $H$. lepturus venom administration

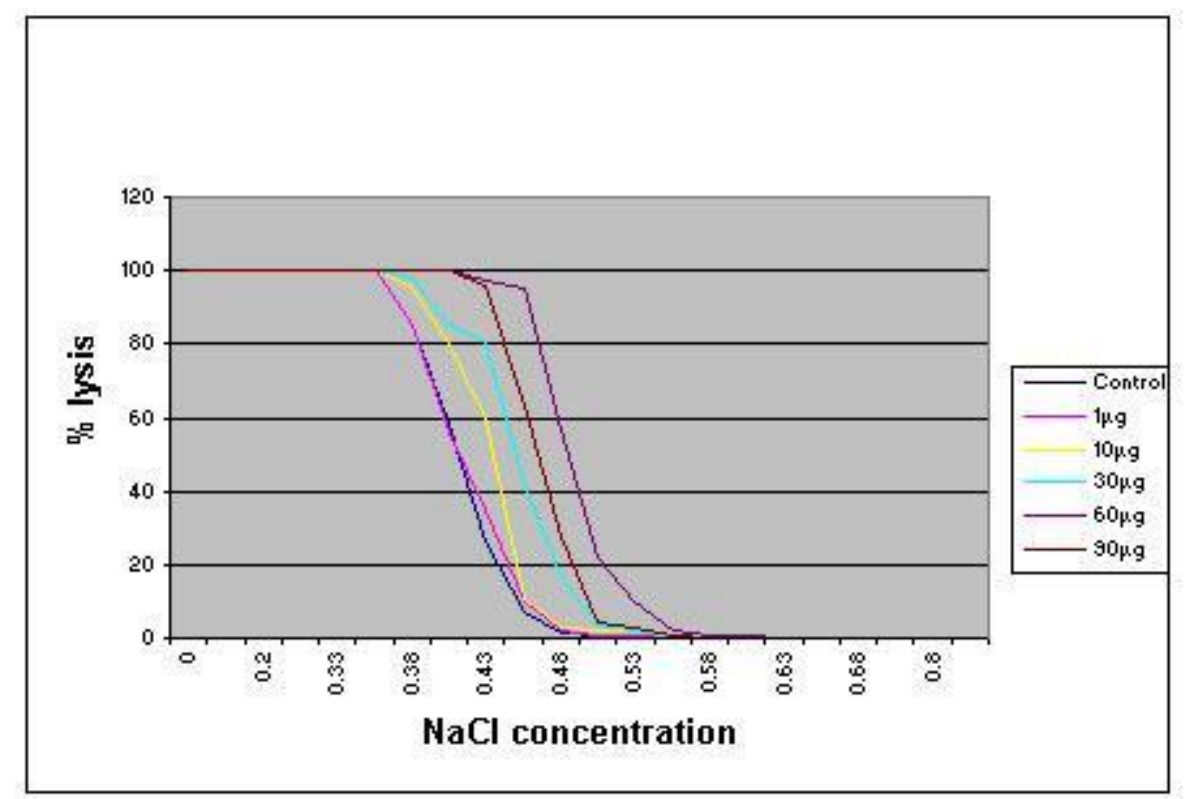

Figure 1. In vitro osmotic fragility of rabbits' red blood cells incubated at increasing concentrations of $\mathrm{NaCl}$ and exposed to various concentrations of $H$. lepturus venom.

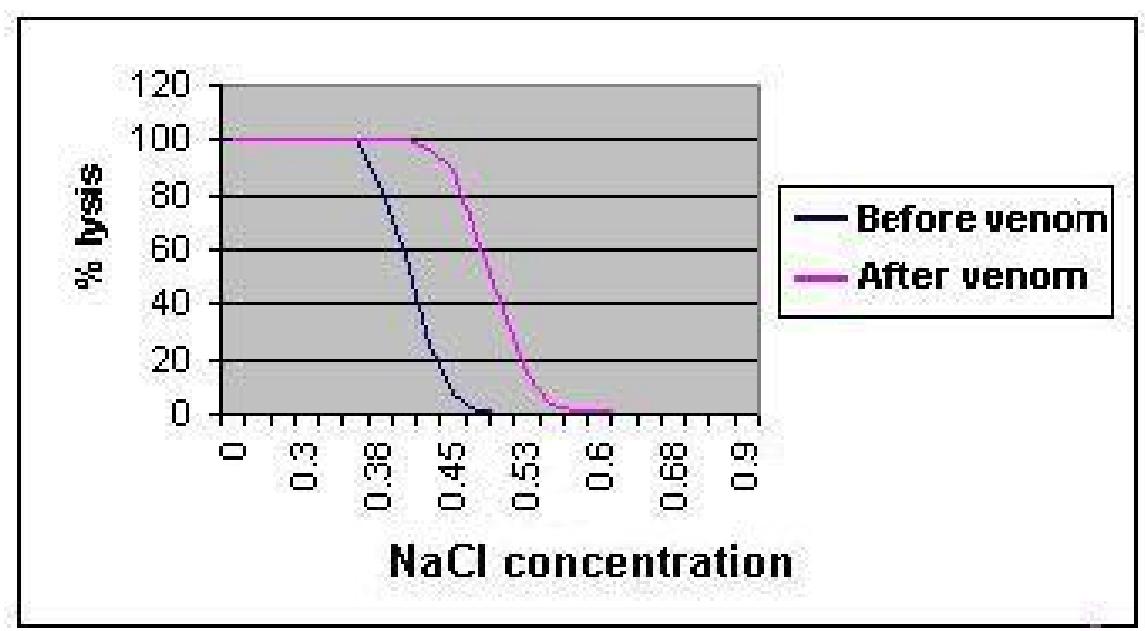

Figure 2. In vivo osmotic fragility of rabbits' red blood cells incubated at increasing concentrations of $\mathrm{NaCl}$ before and after $\mathrm{H}$. lepturus venom injection. 
Table 1. Comparison of various biochemical parameters before and three hours after venom injection into experimental rabbits.

\begin{tabular}{lcccc}
\hline \multicolumn{1}{c}{ Parameters } & & $\begin{array}{c}\text { Before Venom } \\
(\text { Mean } \pm S D)\end{array}$ & $\begin{array}{c}\text { After Venom } \\
\text { (Mean } \pm S D)\end{array}$ & $p$ Value \\
\hline Blood Glucose $(\mathrm{mg})$ & $112 \pm 25$ & $285 \pm 74$ & $\mathrm{p}<0.001$ \\
\hline Aspartate Aminotransferase $(\mu \mathrm{g} / \mathrm{l})(\mathrm{AST})$ & $23.42 \pm 5.9$ & $47.70 \pm 8.50$ & $\mathrm{p}<0.001$ \\
\hline Alanine Aminotransferase $(\mu \mathrm{g} / \mathrm{l})$ & $(\mathrm{ALT})$ & $26.63 \pm 8.2$ & $73.41 \pm 12.42$ & $\mathrm{p}<0.001$ \\
\hline Alkaline Phosphatase $(\mu \mathrm{g} / \mathrm{l})$ & $(\mathrm{ALP})$ & $121.43 \pm 34.2$ & $173.11 \pm 74.32$ & $\mathrm{NS}$ \\
\hline Lactate Dehydrogenase $(\mu \mathrm{g} / \mathrm{l})$ & $(\mathrm{LDH})$ & $155.54 \pm 17.54$ & $298.34 \pm 29.11$ & $\mathrm{p}<0.001$ \\
\hline Creatine Phosphokinase $(\mu \mathrm{g} / \mathrm{l})$ & $(\mathrm{CPK})$ & $24.45 \pm 8.43$ & $36.23 \pm 10.34$ & $\mathrm{NS}$ \\
\hline
\end{tabular}

NS $=$ Not Significant

\section{DISCUSSION}

Hemiscorpius lepturus is a dangerous scorpion from the south and southwestern part of Iran that causes mortality and morbidity in both children and adults $(1,14)$. Although many scientific reports have clarified the complex mechanism of action of venoms of scorpions of the Buthidae family $(3,4,10,16)$, scarce studies showed the mechanism involved in envenomation by scorpions of the Scorpionidae family, especially $H$. lepturus (14).

Clinical reports have indicated a high hemolysis rate in patients stung by $H$. lepturus (14). In the southwest of Iran, hemolysis due to envenomation by such scorpion is so common that when a patient is suspected of having been stung by a scorpion, the first clinical test is to check the presence of hemoglobin in the patient's urine. An interesting fact in $\mathrm{H}$. lepturus sting is the absence of local pain or the presence of very mild pain at the sting site (14). Many times, absence of local pain can delay treatment.

In the current study, local signs manifested as a local reddish edema of about $2-3 \mathrm{~cm}$ around the injection site between 15 and $20 \mathrm{~min}$ after venom administration. Systemic signs and symptoms (increased heart rate and breathing) were observed $20-35 \mathrm{~min}$ following injection. However, the grading of clinical symptoms was not established in the present study. Clinicians in southwest Iran believe that the distribution rate of $H$. 
lepturus venom is very slow, compared with that of venoms of the Buthidae family; thus, they propose the removal of the local tissue after scorpion sting. The manifestation of systemic signs and symptoms occurred from 30 to $35 \mathrm{~min}$ after venom injection, which is not significantly different from the time of emergence of systemic signs and symptoms in cases of envenomation by other scorpions $(10,17)$. This indicates that the distribution rate of neurotoxins is similar in envenomation by all scorpions including those of the Scorpionidae family. All the animals died within 3$4 \mathrm{~h}$ after having received the venom. This was also similar to the time scorpions of the Buthidae family needed to kill rabbits (18). Thus, removing the skin at the sting site seems to be of no use and may even complicate the treatment.

Increased osmotic fragility of RBCs exposed to scorpion (Mesobuthus tamulus) venom, both in vitro and in vivo, was previously reported (11). In the present study, when RBCs were exposed in vitro to various concentrations of $H$. lepturus venom, there was a highly significant $(p<0.001)$ increase in osmotic fragility. This is an indicator of the venom direct action on RBCs. In vivo results of osmotic fragility also confirmed the increased osmotic fragility of RBCs, which may be due to the presence of an enzyme, like phospholipase A2, in the venom of $H$. lepturus and which may be the cause of hematuria in patients stung by this scorpion species. Whether the action of venom on other tissues is similar to that on RBCs is to be clarified in future studies. Determination of blood sugar levels in the serum of rabbits showed a highly significant increase compared with the levels before venom administration. The levels of CPK, ALT, AST and LDH enzymes also increased after venom injection. All these changes were similar to those observed in experimental animals receiving venom of scorpions of the Buthidae family $(18,19)$. Clinical manifestations of scorpion envenomation appear to be secondary to the activation of both sympathetic and parasympathetic autonomic nervous system $(3,4,10)$. Such manifestations reveal that scorpion envenomation causes an autonomic storm $(6,7,9)$. In the present study, significant hyperglycemia was noticed after $H$. lepturus venom administration, which is quite compatible to the results of other studies $(2,8,12,19)$. The proposed mechanism for this effect included: peripheral and central stimulation of the adrenergic system; activation of $\beta$-receptors with catecholamine and serotonin secretion; blockage of insulin secretion; and insulin resistance $(3,16,19)$. The increase in AST and ALT levels in envenomed animals is in agreement with former 
reports $(12,17)$. Aspartate aminotransferase, as a transferase enzyme, is distributed to all parts of the body but mostly concentrates in the liver and the heart; therefore, the increase in its levels may be attributed to myocardial infarction or hepatic failure. Alanine transferase, acting similarly to AST, is considered a liver-specific enzyme. It increases more and remains longer than AST during hepatic failure or inflammation. Therefore, the increase in AST and ALT levels may be due to a direct action of the venom on the liver and the heart.

Thus, according to the obtained results, the signs and symptoms of envenomation by $H$. lepturus occur through two mechanisms: directly, through the effect of venom components on various cells, including RBCs, causing hemolysis; and indirectly, through the effect of neurotoxins enhancing the release of catecholamines with consequent stimulation of the somatic and autonomic nervous system.

\section{REFERENCES}

1 CHOMAILI B. Scorpionism and its manifestation in Iran. In: Congress of Iranian Association of Pediatric Physicians, I, Ahvaz, Iran, 1993, p 104. Abstracts.

2 EL-ASMAR MF., SOLIMAN SF., ISMAIL M., OSMAN OH. Glycemic effect of venom from the scorpion Buthus minax (L.Koch). Toxicon, 1974, 12, 249-51.

3 GUERON M., LLIA R. And SOFER S. The cardiovascular system after scorpion envenomation: A review. J. Toxicol. Clin. Toxicol., 1992, 30, 245-58.

4 ISMAIL M. The treatment of the scorpion envenoming syndrome: The Saudi experience with serotherapy. Toxicon, 1994, 32, 1019-26.

5 ISMAIL M. The scorpion envenoming syndrome. Toxicon, 1995, 33, 825-58.

6 ISMAIL M., ABD-ELSALAM MA., MORAD AM. Do changes in body temperature following envenomation by the scorpion Leiurus quinquestriatus influence the course of toxicity? Toxicon, 1990, 28, 1265-84.

7 MAZZEI DE DAVILA CA., DAVILA DF., DONIS JH., BELLABARBA GA., VILLARREAL V., BARBOZA JS. Sympathetic nervous system activation, antivenin administration and cardiovascular manifestation of scorpion envenomation. Toxicon, 2002, 40, 1339-46.

8 MURTHY KRK., BILLIMORIA FR., KHOPKAR M., DAVE KN. Acute hyperglycemia and hyperkalaemia in acute myocarditis produced by scorpion (Buthus tamulus) venom injection in dogs. Indian Heart J., 1986, 38, 71-4. 
9 MURTHY KRK., VAKIL AE., YEOLEKAR KE. Insulin administration reverses the metabolic and electrocardiographic changes in acute myocarditis induced by Indian red scorpion (Buthus tamulus) venom in experimental dogs. Indian Heart J., 1990, 42, 35-42.

10 MURTHY KRK., ZARE MA. Effect of Indian red scorpion (Mesobuthus tamulus concanesis, Pocock) venom on thyroxine and triiodothyronine in experimental acute myocarditis and its reversal by species antivenom. Indian J. Exp. Biol., 1998, 36, 1621.

11 MURTHY KRK., ZARE MA. The use of antivenom reverses haematological and osmotic fragility changes of erythrocytes caused by Indian red scorpion. Effect of Indian red scorpion Mesobuthus tamulus concanesis Pocock in experimental envenoming. J. Venom. Anim. Toxins, 2001, 7, 113-38.

12 OMRAN MA., ABDEL-RAHMAN MS. Effect of scorpion Leiurus quinquestriatus $(\mathrm{H}$ and E) venom on the clinical chemistry parameters of the rat. Toxicol. Lett., 1992, 61, 99-109.

13 PARAPART AK., LORENZ PB., PARPART ER., GREGG JR., CHASE AM. The osmotic resistance (fragility) of human red cells. J.Clin.Invest., 1947, 26, 636.

14 RADMANESH M. Clinical study of Hemiscorpion lepturus in Iran. J. Trop. Med. Hyg., 1990, 93, 327-32.

15 REED LH., MUENCH H. A simple method for estimating 50 percent end-points. Am. J. Hyg., 1938, 27, 493.

16 ZARE MA. Scorpion venom poisoning: reversal of biochemical hormonal and pathophysiological disturbances by antivenom and insulin therapy. Bombay: University of Bombay, 1992. 229p. [Thesis - PhD]

17 ZARE MA., JALALI A., JAHROMI EA., VATANPUR H., AKBARY A. Biochemical changes and manifestation of envenomation produced by Odonthobuthus doriae venom in rabbits. J. Venom. Anim. Toxins incl.Trop. Dis., 2006, 12, 67-77.

18 ZARE MA., MURTHY KRK., HAGHNAZARI L. Scorpion venom poisoning in experimental animals. Arch. Inst. Razi, 1994, 44/45, 67-72.

19 ZARE MA., MURTHY KRK., JAFAR MB. Insulin as an effective drug in scorpion venom poisoning. Arch. Inst. Razi, 1994, 44/45, 59-65. 